\title{
Autism Spectrum Disorders (ASD)
}

A utism spectrum Disorders (ASD) are, without doubt, one of the nosological groups that cause the greatest suffering in childhood both for those who are affected with this condition and for those who accompany them (parents, family, caregivers, etc.).

ASD is a group of chronic conditions with onset before the age of three. However, it is believed that in many cases affectation is present at birth or even in the foetal period. Although some children have signs in the first few months of life, others do not become manifest until 24 months of age. In fact, development sometimes appears to be normal until the children are aged 18-24 months, when they stop acquiring new skills and lose those they have already acquired.

Many studies have shown that people with ASD interact, communicate, learn and behave differently from others. Sometimes, however, there appears to be no difference, and sometimes children with ASD show both a 'high level' of functioning in specific areas and a constant need for support in all areas of functioning throughout their lives. Moreover, some people who do not suffer from ASD may also present several symptoms of the disorder.

\section{Description}

According to the 11th edition of the International Classification of Diseases (ICD - 11) sponsored by the World Health Organization (WHO $)^{1}$, ASD is characterized by:

- "Persistent deficits in the ability to initiate and to sustain reciprocal social interaction and social communication and...

- ... a range of restricted, repetitive, and inflexible patterns of behaviour and interests.

- The onset of the disorder occurs during the developmental period, typically in early childhood, but symptoms may not become fully manifest until later, when social demands exceed limited capacities.

- Deficits are sufficiently severe to cause impairment in personal, family, social, educational, occupational or other important areas of functioning and are usually a pervasive feature of the individual's functioning observable in all settings, although they may vary according to social, educational, or other context.

- Individuals along the spectrum exhibit a full range of intellectual functioning and language abilities".

Similarly, the 5th version of the Diagnostic and Statistical Manual (DSM - 5) of the American Psychiatric Association (APA) (American Psychiatric Association, 2014; pp. 50 - 51), states that ASD is characterized by:

A. "Persistent deficits in social communication and social interaction across multiple contexts, as manifested by the following, currently or by history (examples are illustrative, not exhaustive):

1. Deficits in social-emotional reciprocity, ranging, for example, from abnormal social approach and failure of normal back-and-forth conversation; to reduced sharing of interests, emotions, or affect; to failure to initiate or respond to social interactions.

2. Deficits in nonverbal communicative behaviors used for social interaction, ranging, for example, from poorly integrated verbal and nonverbal communi-

\footnotetext{
${ }^{1}$ https://icd.who.int/browse11//-m/en\#/http://id.who.int/icd/entity/437815624
} 
cation; to abnormalities in eye contact and body language or deficits in understanding and use of gestures; to a total lack of facial expressions and nonverbal communication.

3. Deficits in developing, maintaining, and understanding relationships, ranging, for example, from difficulties adjusting behavior to suit various social contexts; to difficulties in sharing imaginative play or in making friends; to absence of interest in peers.

Severity is based on social communication impairments and restricted, repetitive patterns of behaviour [...].

B. Restricted, repetitive patterns of behavior, interests, or activities, as manifested by at least two of the following, currently or by history (examples are illustrative, not exhaustive):

1. Stereotyped or repetitive motor movements, use of objects, or speech (e.g., simple motor stereotypes, lining up toys or flipping objects, echolalia, idiosyncratic phrases).

2. Insistence on sameness, inflexible adherence to routines, or ritualized patterns of verbal or nonverbal behavior (e.g., extreme distress at small changes, difficulties with transitions, rigid thinking patterns, greeting rituals, need to take same route or eat same food every day).

3. Highly restricted, fixated interests that are abnormal in intensity or focus (e.g., strong attachment to or preoccupation with unusual objects, excessively circumscribed or perseverative interests).

4. Hyper- or hyporeactivity to sensory input or unusual interest in sensory aspects of the environment (e.g., apparent indifference to pain/temperature, adverse response to specific sounds or textures, excessive smelling or touching of objects, visual fascination with lights or movement.

Severity is based on social communication impairments and restricted, repetitive patterns of behavior.

C. Symptoms must be present in the early developmental period (but may not become fully manifest until social demands exceed limited capacities or may be masked by learned strategies in later life).

D. Symptoms cause clinically significant impairment in social, occupational, or other important areas of current functioning.

E. These disturbances are not better explained by intellectual disability (intellectual developmental disorder) or global developmental delay. Intellectual disability and autism spectrum disorder frequently co-occur; to make comorbid diagnoses of autism spectrum disorder and intellectual disability, social communication should be below that expected for general developmental level.

Note A: Individuals with a well-established DSM-IV diagnosis of autistic disorder, Asperger's disorder, or pervasive developmental disorder not otherwise specified should be given the diagnosis of autism spectrum disorder. Individuals who have marked deficits in social communication, but whose symptoms do not otherwise meet criteria for autism spectrum disorder, should be evaluated for social (pragmatic) communication disorder.

Not surprisingly, both texts use practically the same terms and phrases, to the extent that an attempt has been made to converge the two definitions (American Psychiatric Association, 2014; pp. 11 - 12). What is significant, as noted in Note A of DSM-5, is the dilution of certain heterogeneous conditions that in the previous edition (American Psychiatric Association, 
2002; 79-97) were considered under the heading of Pervasive Developmental Disorders (PDD), which includes Autistic Disorder, Disintegrative Childhood Disorder, Asperger's syndrome and Pervasive Developmental Disorder Not Otherwise Specified.

\section{Epidemiology}

A recent study carried out in the European Union on a population of over 600,000 children (European Parliament, 2018; p. 3) estimated that, according to ASD's DSM- 5 diagnostic criteria, in the child population between 7 and 9 years of age, the prevalence of ASD in Europe was 11.2 per 1,000 children ( 1 in 89 , i.e., $1.12 \%)^{2}$ with an estimated global prevalence ranging from 4.4 to 19.7 per 1,000 children depending on the participating country.

Another study with similar characteristics (Baio, Wiggins, Christensen, \& al., 2018) conducted in 2014 on a population of over 300,000 children aged 8 years old in the United States indicated a prevalence of 16.8 per 1,000 children (i.e. 1 in 59, or 1.7\%), with an estimated global prevalence ranging from 13.1 to 29.3 per 1,000 children depending on the participating centre. This figure is a significant increase compared to previous periods and confirms the trend that shows an increase in the number of cases diagnosed from 1 in $150(0.67 \%)$ between 2000 and 2002 to 1 in 68 (1.47\%) between 2010 and 2012-, even though the estimates of ASD prevalence were probably lower after the diagnostic criteria were changed from DSM - IV - TR to DSM - 5 (Maenner et al., 2014).

As we see later, early detection is of paramount importance when planning therapeutic interventions. According to the results of the European study, which match those of the American one, "Diagnosis is still a big problem in Europe, and more resources are needed to facilitate early detection". Therefore, although the first concerns regarding autism arise, on average, at 25.3 months of life, parents already perceive a developmental problem before the first year of life and detect alterations in social and communicative skills and in fine psychomotor functioning at as early as 6 months of age. Paradoxically, diagnosis takes place on average 19 months later, at around 44.4 months of life! This alarming fact is not surprising since just over two thirds of parents indicate delays of more than 6 months in accessing diagnostic services.

The American study also indicates that racial differences are being reduced, as the rates of ASD prevalence for African Americans and Hispanics are beginning to match those of the white population. With regard to distribution by sex, the boy-girl ratio of $4: 1$ has remained stable over time.

The economic burden caused by ASD, according to the above European study ${ }^{3}$, is also significant, both in terms of Disability-Adjusted Life Years ${ }^{4}$ (DALYs), with a total of 207,771 DALYs (153,153 for men and 54,618 for women), and in terms of economic cost per individual within 6 months, where figures range from $€ 797$ in Romania to $€ 11,189$ in Denmark. Expenditure on special education resources tops the list, followed by tutored support. Some health and welfare services are a significant component of the costs directly paid by individuals with ASD and their caregivers. In the same period, lost productivity costs by caregivers range from $€ 307.70$ in Poland to $€ 4,467.40$ in Austria. Cost studies in the United States provide similar figures, which highlights the fact that the costs of medical care for children with ASD are 4 to 6 times higher than for the same population without ASD (Shimabukuro, Grosse, \& Rice, 2008).

With regard to the economic impact that the implementation of a universal detection system such as the one in Spain - which includes regular primary care medical check-ups for

\footnotetext{
2 There appears to be a miscalculation: the figure indicated in the study is 12.2 per 1,000 ; however, when the calculation is made, the resulting figure is the one indicated in this text.

${ }^{3}$ European Parliament (2018), pp. $4-5$.

${ }^{4}$ The DALYS or DALY is a global measure of burden of disease, expressed as the number of years lost due to illness, disability or premature death. Developed in 1990 to compare the overall health and life expectancy of different countries, its use is increasingly common in public health and health impact assessment.
} 
children under two years of age and the use of M-ChAT (Modified Checklist for Autism in Toddlers) - would have in different European countries, the annual cost would range from $€ 43,000$ in Iceland to $€ 5$ million in France 5 .

\section{Aetiology}

There is no single or identifiable cause for the heterogeneity of clinical symptoms included under the denomination of ASD. The factors that can result in ASD may be environmental, biological or genetic, with a combination of these factors being the most likely cause. In most cases, however, the causes are unknown.

With regard to environmental factors, evidence seems to show that the perinatal period is critical to the development of ASD, to the extent that a whole series of events may occur, including "abnormal presentation, umbilical-cord complications, fetal distress, birth injury or trauma, multiple birth, maternal hemorrhage, summer birth, low birth weight, small for gestational age, congenital malformation, low 5-minute Apgar score, feeding difficulties, meconium aspiration, neonatal anemia, $A B O$ or Rh incompatibility, and hyperbilirubinemia" even though "There is insufficient evidence to implicate any 1 perinatal or neonatal factor in autism etiology" (Gardener, Spiegelman, \& Buka, 2011).

With regard to biological factors, an increased risk of ASD has been associated with intrauterine exposure to drugs such as valproic acid (which is used in the maintenance treatment for bipolar disorder as well as in the stabilization of several types of epilepsy) and thalidomide (which was initially used for its antiemetic action and is currently indicated for the treatment of myeloma). On the other hand, the association between ASD and vaccines has been ruled out (Demicheli, Rivetti, Debalini, \& Di Pietrantonj, 2012; PP. 19) ${ }^{6}$. This is a controversy that arises regularly in the media to feed one of the arguments of supporters of the Antivaccine movement ${ }^{7}$.

Finally, with regard to genetic factors, studies on populations of identical monozygotic twins indicate that the probability that one of the two siblings is affected when the other one also is, is roughly $36-95 \%$, while the range is clearly lower $(0-31 \%)$ for non-identical twins. Moreover, the possibility of having a second son affected by ASD is $2-18 \%$. This risk also increases in children of older parents ${ }^{8}$. However, although there is an identifiable genetic cause in up to $25 \%$ of cases (e.g. fragile $X$ syndrome or tuberous sclerosis), a review of genetic studies (Huguet, EY, \& Bourgeron, 2013) revealed "the genetic landscape of ASDs to be highly heterogeneous, with different types of genetic abnormalities located on almost all chromosomes with varying levels of penetrance". The review also noted "the identification of a large number of causative genes that converge in common pathways" and concluded that "The clinical outcomes associated with the causative genes exceed the boundaries of ASD because the same genes associated with ASD [...] are also associated with other neuropsychiatric disorders, such as schizophrenia and bipolar disorder".

\footnotetext{
${ }^{5}$ Op. Cit.

6 "We could assess no significant association between MMR immunization and the following conditions: autism, asthma, leukaemia, hay fever, type 1 diabetes, gait disturbance, Crohn's disease, demyelinating diseases, or bacterial or viral infections. The methodological quality of many of the included studies made it difficult to generalise their results".

7 See: Vaccine Controversy. (2018, 16 November). Wikipedia, The free encyclopedia. Date of consultation: 17:47, February 22, 2019 from

https://es.wikipedia.org/w/index.php?title=Controversia de las vacunas\&oldid=112060065.

${ }^{8}$ https://www.cdc.gov/ncbddd/autism/data.html
} 


\section{Pathophysiology}

\section{Brain functioning}

Anomalies in different areas of the brain have been related to ASD. Increase in brain volume could therefore be an early indicator of autism that precedes the disorder's behavioural onset. Alterations in the cerebellum could explain failures in attention, motor behaviours, associative learning or activation. The intermediate temporal lobe has been involved in the perception and recognition of faces and facial expressions, reward circuits, recognition of the affective meaning of stimuli, the perception of body movements such as direction of gaze, certain cognitive abilities that are important for social perception, imitation, joint attention and the Theory of Mind. Alterations in the ventromedial and dorsolateral prefrontal cortex, Broca's area and the inferior parietal cortex have been detected. One finding that has received most attention in recent times involves mirror neurons (Rizzolatti, FADAM, Welsh, \& Fogassi, 1996):

"In area F5 of the monkey premotor cortex there are neurons that discharge both when the monkey performs an action and when he observes a similar action made by another monkey or by the experimenter. We report here some of the properties of these 'mirror' neurons and we propose that their activity 'represents' the observed action. We posit, then, that this motor representation is at the basis of the understanding of motor events. Finally, on the basis of some recent data showing that, in man, the observation of motor actions activate the posterior part of inferior frontal gyrus, we suggest that the development of the lateral verbal communication system in man derives from a more ancient communication system based on recognition of hand and face gestures".

Later, we will refer to the relationship between mirror neurons and autism through the Theory of Mind (ToM).

\section{Neurotransmitters}

Numerous studies have investigated the role of neurotransmitters in the genesis of ASD. The neurotransmitters that have been most frequently associated with ASD are GABA, glutamate, serotonin, epinephrine and norepinephrine. Other hypotheses, however, involve endogenous opiates and changes in oxytocin transmission. Autoimmune processes have also been held responsible as antibodies against myelin basic protein in children with autism, as have increases in eosinophil and basophil responses mediated by IgE (Cetin, Tunca, Sur, \& Iseri, 2015).

\section{Psychological Theories}

\section{From the Theory of Mind (ToM) to Intersubjectivity}

"In saying that an individual has a theory of mind, we mean that the individual imputes mental states to himself and to others (either to conspecifics or to other species as well). A system of inferences of this kind is properly viewed as a theory, first, because such states are not directly observable, and second, because the system can be used to make predictions, specifically about the behavior of other organisms".

In their article "Does the chimpanzee have theory of mind?", Premack and Woodruff (1978) defined this concept, which comprises the ability to infer purposes or intention, beliefs, thoughts, knowledge, tastes, guesses, pretensions, promises and trust. This concept was later expanded to include the ability to infer emotions and constitute the capacity to reflect on the content of one's own mind and that of others.

Although initially highly suggestive and stimulating when attempting to explain the difficulties inherent to autistic children, the inadequacy of the model led researchers to look for 


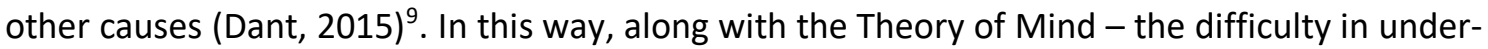
standing the minds of others - other cognitive features that are altered in ASD are Central coherence (the ability to integrate information into context, which is low in opposition to high local perception and detail), Executive dysfunction (alteration of the processes underlying control of the action that aims to achieve a goal, including attentional control, and indicates prefrontal cortex abnormalities), and, according to some authors, Joint attention (monitoring what one and another person are looking at) (Baron-Cohen, 2000).

Approaches based on Intersubjectivity ${ }^{10}$ integrate neurobiological findings that characterize these patients by taking into account observational data on difficulties in people with ASD in the first few months of life while attaching greater importance to problems of sensorymotor processes (Trevarthen and Delafield-Butt, 2013). Approaches based on neurophenomenology ${ }^{11}$ (Gallagher, 2007) assert that two sets of phenomena are established from the neurobiological problems that are presumed to be at the origin of ASD. The first set of phenomena comprises those relating to sensory-motor processes, which affect the establishment of primary intersubjectivity and subsequently secondary intersubjectivity and could account for symptoms such as echolalia, hypersensitivity to stimuli and repetitive and bizarre movements (stereotypes). The second set comprises derivatives of failures in central coherence that, through alterations in perception, affect primary intersubjectivity and, through cognitive alterations, affect secondary intersubjectivity, and could account for symptoms such as a narrower range of interests, the need for routine, alterations in the non-semantic perception of form, and Gestalt problems. Ultimately, alterations in secondary intersubjectivity alter the processes involved in developing the Theory of Mind.

\section{Psychodynamic theory}

Also, from an intersubjective approach, psychoanalytic theories consider that autism represents a primitive defence mechanism - in the sense that it is one of the most ancient filo- and ontogenetic mechanisms - that is put into motion by the invasion of intense anguish experienced in the earliest stages of life. The resulting 'withdrawal from the world' ${ }^{12}$ is devastating to the extent that it compromises the establishment of the necessary bonds that help to structure and develop an individual's psyche. These bonds are then established on the alternation between the most absolute dependence, leading to intense separation anxieties as threatened by the differentiation of the other, and the rejection of any form of relationship at the risk of being invaded by the presence of the other.

\footnotetext{
${ }^{9}$ In his work on the concept of ToM and its relationship with the theory of intersubjectivity, Dant concludes that: "while 'theory of mind' has stimulated research and discussion on autism, the phenomenological understanding of 'intersubjectivity' is more appropriate to trying to understand the difficulties faced by people with autism"(p. 45).

${ }^{10}$ Lecannellier's (2006) conceptualization is useful for defining intersubjectivity in a child's early development. He uses this term to refer to both the affective processes that occur between caregiver and baby in the first years of life and the development of mechanisms of subjective recognition of the other that are structured from these affective encounters. Primary intersubjectivity refers to the temporally and emotionally regulated exchanges observed in early dyadic interactions between the caregiver and the baby during the period between 2 and 9 months that also includes neonatal imitation, protoconversations, and interactive synchrony. For its part, the term secondary intersubjectivity describes those situations that emerge between 9 and 12 months of life in which the baby is able to combine, in interaction with the caregiver, two types of acts: praxic - pointing, showing, giving, offering, taking an object, consecutive manipulation, praxic imitation, regulating the action on the object, resisting, touching the object, extending the hand; and interpersonal - smiling, vocalizing, looking at another's face, extending arms to the caregiver, touching the other, and vocal imitation.

${ }^{11}$ Work to understand the current state of theoretical conceptions about ASD is recommended.

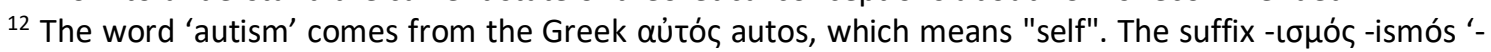
ism' is added to form an abstract noun that denotes this tendency.
} 
Some of the controversies with psychoanalysis arise because of its opponents' ignorance of the concept of complementary series ${ }^{13}$, which infers the exclusion of organic factors in the genesis of clinical manifestations to emphasize, almost exclusively, the importance of the child's relationship with the caregiver. What happens is that the caregiver's good influence can be overwhelmed by problems in biological endowment, whereas good biological endowment cannot, on its own, determine an infant's good psychic evolution if his/her relationship with the caregiver is insufficient or pathological.

\section{Clinical symptoms}

According to the preceding section, the symptomatology of ASD is related to the alteration of sensory processes and central coherence, so the establishment of both primary and secondary intersubjectivity and the development of the Theory of Mind will be affected.

Expected developmental milestones for the child's chronological age must always be considered, bearing in mind that a range of possible variations exists (from precocious to delayed) outside which pathology may be considered. Another characteristic to be evaluated in the child's development is whether it is 'harmonic' in the areas considered, i.e. there is a certain homogeneity in each area, or whether it is 'disharmonic', i.e. areas with significant development, sometimes above expected for the child's age, alternate with others with a deficit or even with no progress at all.

Symptoms are usually described, on the one hand, according to how the areas of communication and social interaction are affected, and, on the other, by the presence of restricted interests. A useful description and grouping of symptoms is the one created below by Johnson (2004):

\section{Possible "Red Flags"}

People with ASD may:

- Flap their hands, rock their body, or spin in circles.

- Have unusual reactions to the way things sound, smell, taste, look, or feel.

- Not respond to their name by 12 months of age.

- Not point at objects to show interest by 14 months.

- Not play 'pretend' games (pretend to 'feed' a doll) by 18 months.

- Avoid eye contact and want to be alone.

- Have delayed speech and language skills.

- Repeat words or phrases over and over (echolalia).

- Give unrelated answers to questions.

- Have trouble understanding other people's feelings or talking about their own feelings.

- Get upset by minor changes.

- Have obsessive interests.

\section{Signs and symptoms related to social interaction}

People with ASD may:

- Not respond to their name by 12 months of age.

- Avoid eye-contact.

\footnotetext{
${ }^{13}$ Since its inception, psychoanalysis has held that every psychic phenomenon - and autism - obeys the laws of complementary series, which state that the origin of every psychic phenomenon is the result of the interaction between the biological endowment of each human being and his or her early experiences. In agreement with modern genetics, Freud called this combination the genetic factor. It is therefore not true to argue that psychoanalysis has denied the importance of biological endowment in normal and pathological psychic phenomena.
} 
- Prefer to play alone.

- Not understand personal space boundaries.

- Not share interests with others.

- Interact only to achieve a desired goal.

- Have apathetic or inadequate facial expressions.

- Not understand the boundaries of personal space.

- Avoid or resist physical contact.

- Not feel the consolation provided by other people when they are distraught.

- Have difficulty understanding other people's feelings and expressing their own.

\section{Signs and symptoms related to communication}

People with ASD may:

- Experience a delay in their speech and language skills.

- Repeat words or phrases constantly (echolalia).

- Use pronominal inversion, i.e. use 'you' instead of 'me' and vice versa.

- Speak in a monotonous, robotic, or treble tone of voice.

- Persevere on a topic of conversation for too long, talking about what they like rather than having a reciprocal conversation with the other person.

- Give answers that are not related to the questions asked.

- Use language in unusual ways:

- Be unable to put words into real sentences.

- Say just one word at a time or repeat the same words or phrases repeatedly.

- Not point or respond when pointed to something.

- Use few or no gestures.

- People with ASD may have difficulty using and understanding gestures, body language, or tone of voice.

- e.g. they may not understand what it means to say goodbye by hand.

- The facial expressions, movements, and gestures of people with ASD may not match what they are saying.

- e.g. they may smile when saying something sad.

- Not play simulation games (e.g. 'feeding' a doll).

- Not understand jokes or sarcasm

Some children with good language skills may:

- Speak like little adults, unable to express themselves as children normally do.

\section{Signs and symptoms that indicate interests and unusual behaviours}

People with ASD may:

- Be very organized.

- Become irritated by small changes.

- Have obsessive interests.

- Need to follow certain routines.

- Form lines with toys or other objects.

- Always play with toys in the same way.

- Show an interest in certain parts of objects (e.g. wheels).

- Flap hands, rock or spin in circles. 
- e.g. spend a lot of time flapping their arms repetitively or rocking from side to side; turn a light on and off or rotate the wheels of a toy car repeatedly. This type of activity is known as auto-stimulation or 'stereotyped behaviours'.

- Need routine:

- A change in the usual routine for a day, like stopping on the way home from school, can be distressing, causing them to 'lose control' and have a 'crisis' or a tantrum, especially if they are in a place they do not know.

\section{Signs and symptoms that may occur in ASD but are not central aspects of the condition People with ASD may display:}

- Hyperactivity/impulsiveness/low levels of concentration

- Tantrums

- Aggressiveness

- Self-harming behaviours

- Unusual eating and sleeping habits

- Mood swings or unusual emotional reactions

- Lack of fear or more fear than expected

- Unusual reactions to sounds, smell, taste, appearance or touch:

- e.g. they may have little or an exaggerated reaction to pain or loud noises; abnormal eating habits, such as limiting their diet to certain foods or eating inedible things like soil or stones

- Problems such as chronic constipation or diarrhoea.

\section{Comorbidity}

These symptoms introduce the issue of comorbidity, i.e. other psychiatric disorders people with ASD have that are not symptoms of ASD. Comorbidity occurs in almost $70 \%$ of patients, which should make us reflect on how international classifications organize diagnoses. In $40 \%$ of cases, two or more psychiatric diagnoses concur in addition to ASD (American Psychiatric Association, 2014).

ASD is often associated with intellectual disability, though almost half of the ASD population have an IQ that is average or above average. It is also very often associated with structural alterations of speech, i.e. the inability to understand or construct grammatically correct sentences. Other conditions that patients with ASD may present jointly are ADHD, developmental disorder of coordination, anxiety disorder, and depressive disorder, etc.

\section{Diagnosis}

As with practically all psychiatric pathologies, the diagnosis of ASD is eminently clinical. As we mentioned earlier, it is usually parents who detect the first signs of alarm, and delays in obtaining a diagnosis imply a crucial loss of time when planning interventions.

In accordance with the proceedings in the Process of comprehensive care for people with Autism Spectrum Disorder (Valencian Ministry of Universal Health and Public Health, 2017), psychomotor development should be monitored during general primary care visits by paediatricians using validated scales such as Haizea-Llevant. In cases of risk, special attention should be paid to the warning signs, and the M-ChAT screening instrument should be completed at 18 months of age.

If ASD is suspected, the paediatrician should send the patient to the Early Assessment Unit for assessment and intervention and to the Child and Adolescent Mental Health Unit (USMIA) for further assessment and confirmation of diagnosis. The USMIA will coordinate care 
among the intervening professionals, including the child psychiatrist, the clinical psychologist, the paediatrician, the neuropaediatrician, and those at the early intervention centre, the child's school, and the social services.

The following evaluation instruments are available:

\section{Screening tests}

- SCQ (Social Communication Questionnaire). This instrument is designed to quickly evaluate the communication and social relations skills of children who may suffer from ASD. It collects the symptoms observed by parents and caregivers to decide whether it is advisable to refer the child for further evaluation.

- M-ChAT-R ${ }^{14}$ (Modified Checklist for Autism in Toddlers - Revised) (Robins, Fein, \& Barton, 2009). This questionnaire is based on 20 questions, three of which are considered critical, answered by observing the behaviour of the children. It is used in many countries to detect autism spectrum disorders in children aged between 18 and 60 months.

- The M-ChAT-R can be administered during a visit to the Healthy Child Care programme or by specialists or other professionals to assess the risk of ASD. The main objective of M-ChAT-R is to achieve maximum sensitivity and detect as many cases of ASD as possible. There is therefore a high rate of false positives, which means that not all children who are given an at-risk rating are diagnosed with ASD.

- Follow-up questions (M-ChAT-R /F) have been developed to solve this problem. Users should be aware that even with this follow-up, a significant number of children who test positive on the M-ChAT-R will not be diagnosed with ASD. However, as these children are at high risk of other developmental or delayed disorders, evaluation is guaranteed for any child whose result is positive. The $M-C h A T-R$ can be evaluated in less than two minutes.

- ADBB (from the French Alarme Détresse Bébé). This systematized infant observation guide is used to detect early withdrawal indicators in infants aged from 2 to 24 months.

- AUTONOMOUS SCALE FOR THE DETECTION OF ASPERGER'S SYNDROME AND HIGHFUNCTIONING AUTISM. This is not a diagnostic test per se, but it enables professionals to objectively verify a possible social disability and decide when it is advisable to consult a specialist in clinical psychology, psychiatry and/or developmental disorders. It is intended for children aged six years old and upwards.

\section{Diagnostic tests}

- ADI-R (Autism Diagnostic Interview - Revised). This semi-structured interview for parents is recommended for children with a developmental level of more than 18 months.

- ADOS (Autism Diagnostic Observation Schedule). This is a semi-structured observational evaluation instrument with four different modules depending on the child's age and language ability. A new version has also been created for children without verbal language (PL-ADOS) as well as ADOS-2, which includes the T-module for evaluating children aged 12 months old and upwards.

- CARS (Childhood Autism Rating Scale). This is an observational instrument for children and adults. The recommended age is 2 years. It is easy to apply and has good sensitivity for discriminating between people with and without autism.

\footnotetext{
${ }^{14}$ https://mchatscreen.com/mchat-rf/translations/
} 
- ACACIA. This instrument, designed by Spanish researchers, evaluates autism in children from 2 years old and upwards. It presents hypothetical situations that trigger basic (instrumental or anticipation) social strategies, enabling observation of important categories of social interaction. It is aimed at children with a mental age of less than 36 months and with few or no competences in functional expressive language.

- IDEA. This instrument assesses the severity and depth of autistic traits in cases of autism spectrum disorders and Asperger's syndrome. It is intended for children aged from 5 years old and upwards.

\section{Differential Diagnosis ${ }^{15}$}

In daily practice it is necessary to make differential diagnoses between ASD and other conditions such as other neurodevelopment disorders, intellectual disabilities, certain sensory deficits and situations of severe emotional negligence. This is not always easy.

\section{Atypical autism/pervasive developmental disorder not specified}

This heading categorizes cases that do not follow all the specific patterns required to diagnose ASD, i.e. the same deficits are presented but not all diagnostic criteria are met. Non-specificity may be due to the pattern of the symptoms, their severity or age of onset. In any case, these patients require the same therapeutic measures. It is not known whether presentation of any of these symptoms implies a different etiopathology.

\section{Asperger's syndrome}

Under Asperger's syndrome are grouped individuals whose intellectual development is good, whose verbal IQ is higher than their non-verbal IQ, and who display proper acquisition of language. This disorder is sometimes called high-performance autism. However, diagnostic criteria in terms of operational definition have been unsatisfactory and do not enable selection of a homogeneous sample. This has led to contradictory results across various studies, which in turn has led to its exclusion as a specific category in international classifications. Due to the absence of any delay in the acquisition of language, diagnosis tends to be made substantially later than for autism. It is not known whether the pattern of neurological deficits in Asperger's syndrome is different from that of ASD.

\section{Rett's syndrome}

This is a pervasive developmental disorder that affects exclusively 1 in every 10,000-15,000 girls and is the only one with a known genetic cause: mutation in the X-linked gene that encodes methyl-CpG binding protein 2 . It is characterized by a relatively normal general and psychomotor development during the first 6-18 months of life, followed by stagnation of development acquisitions and a rapid deterioration in behaviour and mental state, leading to dementia with autistic features in 18 months, including loss of the intentional use of the hands after previous acquisition of the prehensile function, unbalanced ataxia of the trunk and extremities, uncomfortable and unstable gait, and acquired microcephaly. After a prolonged period with a relatively stable mental state for years, other neurological anomalies arise, especially lower extremity spasticity and epilepsy. No specific treatment is currently available, though recent studies in animals suggest that neuronal degeneration can eventually be reversed.

\footnotetext{
${ }^{15}$ Extracted from Rutter et al. (2011), pp. 763-764.
} 


\section{Infantile disintegrative disorder or Heller's syndrome}

This is a very uncommon disorder with a low prevalence rate $(0.2$ per 10,000$)$. It is characterized by apparently normal development during the first two years of life, after which there is loss of receptive and expressive language and motor coordination and the development of faecal and urinary incontinence, social withdrawal, hand stereotypes and simple rituals similar to those observed in autism. Deterioration continues for several months before it reaches a plateau. The condition is often difficult to distinguish from autism combined with intellectual deterioration. In some cases, deterioration progresses with increased motor dysfunction, epileptic seizures and localized neurological deficits. Some cases have been associated with cerebral lipidosis and leukodystrophy. It is unknown whether the condition constitutes an atypical variant of ASD or is a significantly different syndrome.

\section{Landau-Kleffner syndrome or acquired aphasia with epilepsy}

This condition affects children whose development is normal until they acquire a loss of receptive and expressive language together with an onset of epileptic seizures or transient alterations in the electroencephalogram (EEG). Regression may be accompanied by social retraction and behavioural disturbances, while cognitive functioning and non-verbal motor function remain intact. Sometimes, language is regained.

\section{Intellectual disability}

According to numerous studies, up to two-thirds of children with autism also present intellectual disability. On the other hand, approximately half of children with an IQ of less than 50 also show alterations in social communication, stereotyped behaviour and/or developmental disorders of language, symptoms typical of the diagnostic criteria for autism. In daily practice it is not always easy to determine whether a child presents a 'pure' intellectual disability or an ASD-related intellectual deficit.

\section{Receptive-expressive language disorders}

Delay in the acquisition of language is a frequent reason for the initial referral of children with ASD. Unlike children with ASD, children with a language-development disorder have less serious alterations and usually show a non-verbal IQ that is within normal range. Many of these children may present symptoms that overlap with ASD and some of them match the description for semantic-pragmatic disorder, i.e. they present problems with the social communicative aspects of conversation. Children with receptive-expressive language disorder may show immediate echolalia, severe deterioration in social relations and a limitation in symbolic play. Unlike ASD children, they seldom show restricted or stereotyped behaviours and display no alteration in non-verbal communication.

\section{Sensory deficits}

Another common reason for parental referral of a child with ASD is that the child may be deaf since he/she does not react to his/her name or to intense noises. Similarly, the absence of visual contact displayed by some autistic children from an early age, with their gaze lost in space or fixed on some object, make parents believe their child could be blind. Thorough exploration that includes auditory and visual evoked potentials should be carried out.

\section{Emotional negligence}

Children who have been institutionalized in early childhood (for example, in orphanages) and have been unable to establish a stable bond with any caregiver suffer the consequences of 
such emotional deprivation. These children may show a delay in the acquisition of language and display abnormal social behaviour and restricted interests. Although the clinical picture is very similar to that of ASD, there is usually more social reciprocity. The outcome is also different as disinhibition and restricted interests predominate in latency.

\section{Other psychiatric conditions}

Sometimes differential diagnosis must be considered with other psychiatric conditions that have similar symptoms: for example, with Attention Deficit Hyperactivity Disorder (ADHD) due to impulsivity, hyperactivity, or lack of attention; with Obsessive-Compulsive Disorder (OCD) due to the presence of rituals and perseverance; and with schizoid personality disorder due to social retraction and peculiarities in social interaction.

\section{Withdrawal behaviour in toddlers}

It is important to detect withdrawal behaviour in toddlers because of its implications for prognosis, especially with regard to how the condition will evolve depending on the intervention, and the underlying cause. In this context, it is important to note that social withdrawal is a constant element in conditions such as autism, childhood depression, sensory deficits, developmental delays, disorganized attachment, and chronic pain, etc. This condition may also appear in situations involving lack of self-regulation, anxiety or post-traumatic stress disorders (Guédeney \& Vermillard, 2004).

\section{Treatment $^{16}$}

The most effective results stem from intensive interventions at an early age. The main objectives of treatment are:

1. To facilitate and stimulate the normal development of cognition, language and socialization as much as possible.

2. To decrease autism-related maladaptive behaviours such as rigidity, stereotypes and inflexibility.

3. To reduce or even eliminate specific maladaptive behaviours such as hyperactivity, irritability and impulsivity.

4. To relieve stress and family burden.

Treatment must be individualized (adapted to each individual patient) and must be multidisciplinary in its design and execution (it must include parents, family, school staff and health workers). There must be objective goals and symptoms to work on, usually with behavioural therapies aimed at controlling unwanted symptoms, promoting social interactions, and increasing the patient's self-confidence. Regular supervision of behaviour adjustment, adaptation skills, academic performance, social interactions, communication and interaction with family and peers is needed. Also, when it is needed, medication should be prescribed.

Some of the modes of intervention used to treat ASD are discussed briefly below.

\section{Psychological treatment Early intervention}

This intervention should begin as soon as possible. It should be intensive (at least $20 \mathrm{hrs} /$ week in individual sessions with the child) and it should involve, instruct and support the parents to manage their relationship with their child. It should include several modules and training plans that stimulate the social and communicative functioning of the child in a development-

\footnotetext{
${ }^{16}$ Prepared from Op. Cit. pp. $767-769$.
} 
oriented way and should include systematic instructions on objectives based on applied behavioural analysis ( $A B A)$. It should be possible to generalize the skills acquired to other environments of everyday life.

\section{Parental Work}

One of the complaints parents or caregivers usually make is that they are not given sufficient information. Although almost $50 \%$ of families indicate that professionals have spoken to them about their child's specific needs, up to $20 \%$ of families complain that they did not receive any information at the time of diagnosis, which is extremely serious given the consequences of ASD. It is therefore necessary to provide parents with specific training on clinical features, discuss their feelings of uncertainty regarding outcome, their anxieties about whether right or wrong decisions are being made, their internal discomfort and their feelings of guilt (for themselves or their child) derived from the cessation of personal activities, limited social relations, lack of understanding from others, social stigma, etc. The consequences of a lack of collaboration among the various professionals involved should also be evaluated.

\section{Educational Treatment}

Children with ASD require extra individual attention with a highly structured approach and special education programmes, whether in the context of "Aulas CyL" (Communication and Language) or in a special education school. In the first case, the educator-to-child ratio should be 1:1, depending on the child's level of disability. It is extremely important to ensure continuity of people, classroom and even the time when these activities are carried out. These programmes should also involve training and learning techniques for parents, who should act as co-therapists, in common programmes synchronized with the educational institution.

\section{Pharmacological Treatment}

Pharmacological treatment has not been shown to influence the central symptoms of ASD. However, it is worth considering when dealing with comorbid symptoms that do not respond to behavioural interventions, such as aggressive and self-harm behaviours, temper tantrums, irritability, hyperactivity, rigidity, anxiety, and sleep problems, etc. Various pharmacological groups could therefore be employed, including typical and atypical antipsychotics, mood stabilizers/antiepileptics, antidepressants, psychostimulants, $\alpha 2$-adrenergic agonists and $\beta$ blockers, and melatonin.

More often than for other psychiatric conditions in childhood and adolescence, parents of children with ASD tend to seek complementary and alternative medical treatments for both core and co-morbid symptoms. It is important to respect the family's opinions, critically discuss the results and risk-benefit of those treatments, advise the family on treatments with and without evidence of support, and help determine which treatments are useful by gathering clinical outcome data. Ineffective treatments or treatments whose efficacy has not been proven include secretin administration, sensory integration therapy, mega-doses of vitamin B6 and magnesium, gluten-and casein-free diets, essential fatty acids, treatments, the Son-Rise programme, cranial osteopathy and the administration of hypochlorite (lye).

\section{Outcome and prognosis}

The outcome of ASD is highly variable: $1-10 \%$ have a good prognosis in adult life, i.e. they are able to maintain stable work and form a family, though this percentage depends on the initial diagnosis, an executive IQ $>70$, and the acquisition of verbal communicative language at five years of age. The evolution of intellectual development varies, however: $20 \%$ of patients will 
have an intelligence that falls within the limits of normality; $30 \%$ will suffer mild-to-moderate mental retardation; and up to $50 \%$ will suffer severe-to-deep mental retardation.

All in all, prognosis is not good: roughly $60-66 \%$ of cases will evolve towards severe deficits with a lack of social progress and autonomy. Some cases will have very bad prognosis and will be unable to have any kind of autonomous existence.

Adolescence, and the transformations it implies at all levels (brain development, hormonal readjustment, completion of psychological individuation), leads to a series of dramatic changes, including a worsening of the mental state (temporary in $30 \%$ of cases and permanent in $22 \%$ ). Hyperactivity, destructiveness, loss of language skills preserved to date, intellectual deterioration, epilepsy (of the grand-mal type in up to a third of cases), self-harming and suicidal behaviour can all appear. In fact, suicidal behaviour and epilepsy are the two main causes of the rise in premature mortality in these patients (they occur 18 to 30 years earlier than in people without ASD).

A follow-up study (Hutton, Goode, Murphy, le Couteur, \& Rutter, 2008) of 135 patients aged up to 21 years and diagnosed with ASD had a low dropout rate and collected clinical information via systematic interviews. This study found that $16 \%$ of participants ( 22 cases) developed a new psychiatric disorder that did not simply involve a deterioration in pre-existing autistic features: five patients developed OCD or catatonia; eight patients developed affective disorders with marked obsessive symptoms; three developed complex affective disorders; four developed specific affective disorders; one developed bipolar disorder, and one developed acute anxiety complicated by alcohol abuse. Significantly, there was no case of schizophrenia, though some studies of autistic individuals have indicated the presence of isolated psychotic symptoms in their evolution, including hallucinations and delusional ideas.

\section{Bibliography}

American Psychiatric Association. (2002). DSM-IV-TR: Manual Diagnóstico y Estadístico de los Trastornos Mentales, Texto Revisado. Biblioteca del DSM-IV-TR. Barcelona: Masson.

American Psychiatric Association. (2014). Manual diagnóstico y estadístico de los trastornos mentales (DSM-5 $\left.{ }^{\circledR}\right)$. (Asociación Americana de Psiquiatría, Ed.), Médica Panamericana $\left(5^{\mathrm{a}}\right)$. Arlington: Editorial Médica Panamericana.

Baio, J., Wiggins, L., Christensen, D. L., \& Al., E. (2018). Prevalence of Autism Spectrum Disorder Among Children Aged 8 Years-Autism and Developmental Disabilities Monitoring Network, 11 Sites, United States, 2014 Surveillance Summaries Centers for Disease Control and Prevention MMWR Editorial and Production Staf. MMWR Surveill Summ., 67(6), 1-23. https://doi.org/10.15585/mmwr.ss6706a1

Baron-Cohen, S. (2000). Theory of mind and autism: A review. In Autism (Vol. 23, pp. 169-184). Academic Press. https://doi.org/https://doi.org/10.1016/S0074-7750(00)80010-5

Cetin, F. H., Tunca, H., Güney, E., \& Iseri, E. (2015). Neurotransmitter Systems in Autism Spectrum Disorder. In Autism Spectrum Disorder - Recent Advances (pp. 15-30). https://doi.org/10.5772/59122

Conselleria de Sanitat Universal i Salut Pública. (2017). Proceso de Atención Integral a las personas con trastorno del espectro autista - TEA $\left(1^{\mathrm{a}}\right)$. Valencia: Generalitat Valenciana.

Dant, T. (2015). In two minds: Theory of Mind, intersubjectivity, and autism. Theory \& Psychology, 25(1), 45-62. https://doi.org/10.1177/0959354314556526

Demicheli, V., Rivetti, A., Debalini, M. G., \& Di Pietrantonj, C. (2012). Vaccines for measles, mumps and rubella in children. Cochrane Database of Systematic Review. John Wiley \& Sons, Ltd. https://doi.org/10.1002/ebch.1948

European Parlament. (2018). Autism Spectrum Disorders in the European Union (ASDEU). Executive summary. 
Gallagher, S. (2007). Understanding Interpersonal Problems in Autism: Interaction Theory as An Alternative to Theory of Mind. Philosophy, Psychiatry, \& Psychology, 11(3), 199-217. https://doi.org/10.1353/ppp.2004.0063

Gardener, H., Spiegelman, D., \& Buka, S. L. (2011). Perinatal and Neonatal Risk Factors for Autism: A Comprehensive Meta-analysis. Pediatrics, 128(2), 344-355. https://doi.org/10.1542/peds.2010-1036

Guédeney, A., \& Vermillard, M. (2004). L'échelle ADBB : intérêt en recherche et en clinique de l'évaluation du comportement de retrait relationnel du jeune enfant. Médecine \& Enfance, (juin), 367-371.

Huguet, G., Ey, E., \& Bourgeron, T. (2013). The genetic landscape of autism spectrum disorders. The Annual Review of Genomics and Human Genetics, 14(1), 191-213. https://doi.org/10.1111/dmcn.12278

Hutton, J., Goode, S., Murphy, M., Le Couteur, A., \& Rutter, M. (2008). New-onset psychiatric disorders in individuals with autism. Autism, 12(4), 373-390. https://doi.org/10.1177/1362361308091650

Johnson, C. (2004). Early Clinical Characteristics of Children with Autism. In V. Gupta (Ed.), Autistic Spectrum Disorders in Children (pp. 85-123). New York: Marcel Dekker, Inc. Retrieved from https://www.cdc.gov/ncbddd/spanish/autism/signs.html

Maenner, M. J., Rice, C. E., Arneson, C. L., Cunniff, C., Schieve, L. A., Carpenter, L. A., ... Durkin, M. S. (2014). Potential Impact of DSM-5 Criteria on Autism Spectrum Disorder Prevalence $\begin{array}{llll}\text { Estimates. JAMA } & \text { Psychiatry, }\end{array}$ https://doi.org/10.1001/jamapsychiatry.2013.3893

Premack, D., \& Woodruff, G. (1978). Does the chimpanzee have a theory of mind? The Behavioral and Brain Sciences, (4), 515-526.

Rizzolatti, G., Fadiga, L., Gallese, V., \& Fogassi, L. (1996). Premotor cortex and the recognition of motor actions. Cognitive brain research, 3(2), 131-141. https://doi.org/10.1016/09266410(95)00038-0

Robins, D., Fein, D., \& Barton, M. (2009). Cuestionario M-CHAT Revisado de Detección del Autismo en Niños Pequeños con Entrevista de Seguimiento (M-CHAT-R/F) ${ }^{T M}$.

Rutter, M., Bishop, D., Pine, D., Scott, S., Stevenson, J., Taylor, E., \& Thapar, A. (Eds.). (2011). Rutter's child and adolescent psychiatry (5th ed.). Oxford: Blackwell Publishing.

Shimabukuro, T. T., Grosse, S. D., \& Rice, C. (2008). Medical Expenditures for Children with an Autism Spectrum Disorder in a Privately Insured Population. Journal of Autism and Developmental Disorders, 38(3), 546-552. https://doi.org/10.1007/s10803-007-0424-y

Trevarthen, C., \& Delafield-Butt, J. T. (2013). Autism as a developmental disorder in intentional movement and affective engagement. Frontiers in Integrative Neuroscience, 7 (March 2014). https://doi.org/10.3389/fnint.2013.00049 